\title{
Bromierte Flammschutzmittel in Elektroaltgeräten: Untersu- chung der Brom-Konzentration nach Kunststofftypen und Gerätekategorien mittels Röntgenfluoreszenzanalyse
}

\author{
Aleksander Jandric · Florian Part · Niklas Fink · Marion Huber-Humer · Stefan Salhofer · Christian Zafiu
}

Online publiziert: 18. November 2019

(c) Der/die Autor(en) 2019

Zusammenfassung Kunststoffe in Elektroaltgeräten (EAG) enthalten häufig bromierte Flammschutzmittel (BFR), die unbeabsichtigt freigesetzt und somit zu negativen Umwelt- und Gesundheitsauswirkungen führen können. Zusätzlich vermindern BFR die Qualität von Rezyklaten, wobei Grenzwerte gemäß RoHS-Richtlinie (1000 ppm je BRFTyp) und Abfallbehandlungspflichtenverordnung (AbfBPV) (2000 ppm Gesamtbromgehalt) eingehalten werden müssen. Für diese Studie wurden aus 11 unterschiedlichen EAG-Kategorien 467 Geräte untersucht, aus welchen wiederum insgesamt 1008 Kunststoffproben entnommen wurden. Die Kunststofftypen wurden mittels visuellem Screening des Recycling Codes sowie mittels FTIR-Spektroskopie bestimmt. Die portable Röntgenfluoreszenzanalyse (RFA) diente zur Bestimmung des Gesamtbromgehalts. In den untersuchten EAGs wurden insgesamt 27 verschiedene Kunststofftypen identifiziert, wobei Acrylnitril-ButadienStyrol-Copolymer (ABS) der häufigste Typ ist. Die Messungen mittels RFA haben ergeben, dass in $33 \%$ aller Proben Brom nachgewiesen werden konnte. Der durchschnittliche Bromgehalt betrug ca. $240 \mathrm{ppm}$. Der RoHS-Grenzwert wurde in $18 \%$ aller Proben aus Netzteilen von Druckern, $10 \%$ aus PC-Gehäusen und $9 \%$ aus Flatscreen-Monitoren überschritten. Elektrische Zahnbürsten, Mobiltelefone (ohne Touchscreens), Smartphones und diverse Kleingerä-

DI A. Jandric .

DI Dr. F. Part (四) DI N. Fink .

Univ.-Prof. DI Dr. M. Huber-Humer .

ao. Univ.-Prof. DI Dr. S. Salhofer .

Mag. Dr. C. Zafiu

Department für

Wasser-Atmosphäre-Umwelt, Institut

für Abfallwirtschaft (ABF-BOKU),

Universität für Bodenkultur Wien,

Muthgasse 107, 1190 Wien, Österreich

florian.part@boku.ac.at ten überschritten weder den RoHS-, noch den AbfBPV-Grenzwert. Kunststoffbauteile aus diesen brom-freien EAG würden sich somit besser für ihre Rezyklierung eignen. Da die RFA eine kostengünstige und sehr schnelle Analysemethode ist, würde sie sich dazu eignen, Kunststoffe mit hohem Bromgehalt direkt am Anfallort auszuschließen.

Schlüsselwörter ElektroaltgeräteRecycling - Altkunststoffe - Bromierte Flammschutzmittel · Persistente organische Schadstoffe · Portable Röntgenfluoreszenzanalyse

Brominated flame retardants in WEEE: examination of the bromine concentration by plastic types and device categories using $\mathrm{X}$-ray fluorescence

Abstract Plastics in waste electrical and electronic equipment (WEEE) often contain brominated flame retardants (BFRs), which can be unintentionally released and can have a negative impact on the environment and health. In addition, BFR reduce the quality of recycled materials, whereby limit values according to the RoHS Directive (1000 ppm per BRF type) and the Ordinance on Waste Treatment Obligations (AbfBPV) (2000 ppm total bromine content) must be monitored. For this study, 467 devices were selected from 11 different WEEE categories, from which a total of 1008 plastic samples were taken. The plastic types were identified using visual screening of the recycling code and FTIR spectroscopy. Portable $\mathrm{X}$-ray fluorescence (pXRF) was used to determine the total bromine content. In the WEEE analysed, 27 different plastic types were identified, with acrylonitrile butadiene styrene (ABS) being the most common type (42\%). The XRF measurements showed that bromine was detected in $33 \%$ of all samples. The average bromine content was approx. $240 \mathrm{ppm}$. The RoHS limit value was exceeded in $18 \%$ of all samples from printer power supply units, $10 \%$ from PC housings, and 9\% from flat screen monitors. Neither the RoHS nor the AbfBPV limit value was exceeded in electric toothbrushes, mobile phones (without touch screens), smartphones and various small devices. Such WEEE plastics would therefore be better suited for recycling. As XRF is a cost-effective and very fast analytical method, it could become suitable for excluding plastics with a high bromine content directly at the point of production.

Keywords WEEE recycling · Plastic wastes - Brominated flame retardants . Persistent organic pollutants . Handheld X-ray fluorescence

\section{Einleitung}

Elektroaltgeräte (EAG) haben eine komplexe Zusammensetzung. Sie bestehen im Wesentlichen aus Metallen, Halbund Übergangsmetallen sowie aus organischen Bestandteilen wie Kunststoffen. Ein Anteil dieser Materialien sind wertvolle Sekundärrohstoffe, wie Kupfer, Eisen oder Aluminium, die durch Recyclingprozesse wirtschaftlich wiedergewonnen werden können. Kunststoffe werden fast ausschließlich als Ersatzbrennstoffe eingesetzt, während nicht-metallische, anorganische Materialien (z.B. Keramik, Glas etc.) als inerte Störstoffe bezeichnet und deshalb deponiert werden. Die geringen Recyclingquoten von Kunststoffen lassen sich vorwiegend damit erklären, dass die Zusammensetzung von Kunststoffen sehr heterogen sein kann und die Auftrennung in sortenreine Kunststoffarten somit sehr aufwendig ist. Je nach Gerätekategorie beträgt der Kunststoffanteil zwischen 15 und 45 Gew.-\%, wobei EAG aus bis zu 30 unterschiedlichen Kunststoffty- 
pen bestehen können. Am häufigsten kommen in EAG Thermoplaste vor, wie Acrylnitril-Butadien-Styrol-Copolymer (ABS) (30\%), hochschlagfestes Polystyrol (HIPS) (25\%), Polyamid (PA) (10\%), Polycarbonat (PC)/ABS Blends (9\%) und Polypropylen (PP) (8\%) (Achilias and Antonakou 2015; Stenvall et al. 2013). Die stoffliche Verwertung von EAG-Kunststoffen wird zusätzlich durch den Einsatz von halogenierten bzw. bromierten Flammschutzmittel (kurz FSM) (engl. brominated flame retardants, kurz BFR) erschwert.

BFR werden in Elektrogeräten eingesetzt, um die technischen Anforderungen hinsichtlich Entflammbarkeit zu gewährleisten, da stromführende Bauteile Wärme entwickeln können und von einer grundsätzlichen Brandgefahr auszugehen ist. Bei der Kunststoffherstellung werden BFR entweder "additiv" während des Polymerisation (Buczko et al. 2014; Zhang et al. 2010), oder „reaktiv“ während der Extrusion (Beach et al. 2017) eingearbeitet und somit im Kunststoff dispergiert. Additive BFR, wie Polybromierte Diphenylether (PBDE) oder 1,2,5,6,9,10-Hexabromcyclododecan (HBCD), werden im Herstellungsprozess in die Polymermatrix eingemischt. Diese Additive können jedoch Kunststoffe ungewollt erweichen und in weiterer Folge können diese BFR leichter aus dem Polymer diffundieren. Reaktive BFR, wie Tetrabrombisphenol A (TBBPA), werden chemisch mittels einer kovalenten Bindung in die Polymermatrix eingebunden, womit eine ungewollte Freisetzung stark verlangsamt bzw. verhindert werden kann.

Da zahlreichen BFR human- und ökotoxikologische Wirkungen nachgewiesen wurden (z. B. hormonaktiv bzw. endokrin wirksam) (Hahladakis et al. 2018; Kim et al. 2014), wurden beispielsweise das Inverkehrbringen sowie die Verwendung von PentaBDE und OctaBDE durch die Richtlinie 2003/11/EG in der EU verboten. Der Umgang und die Entsorgung von BFR-haltigen Produkten bzw. Abfällen werden durch die REACH-Verordnung (EC 1907/2006; engl. Registration, Evaluation, Authorisation and Restriction of Chemicals) und RoHS-Richtlinie 2011/65/EU (engl. Restriction of Hazardous Substances) geregelt. Beide EU-Regularien limitieren die erlaubten Konzentrationen bestimmter BFR - i.e. Polybromierte Biphenyle (PBB) und Polybromierte Diphenylether (PBDE) sind mit jeweils $1000 \mathrm{ppm}$ in einem homogenen Ma- terial beschränkt. Das bedeutet, dass Kunststoffe, die mehr als 1000 ppm von einem dieser beiden BFR-Typen enthalten, als gefährliche Abfälle eingestuft und somit nicht recycelt werden dürfen. Da Kunststoffe aus EAG potenziell BFR (über dem RoHS-Grenzwert) enthalten und zusätzlich die Sekundärmärkte für solche Fraktionen generell sehr schwach sind, werden EAG-Kunststoffe in der österreichischen Praxis kaum recycelt, sondern vorwiegend thermisch verwertet. Des Weiteren ist die Abfallcharakterisierung bzw. das BFR-Monitoring in EAG-Kunststoffen sehr zeit- und kostenintensiv, da genau genommen nicht nur die BromKonzentration bestimmt, sondern auch das bromierte Molekül beispielsweise mittels gaschromatischer bzw. massenspektrometrischer Methoden spezifiziert werden müsste. In diesem Zusammenhang muss erwähnt werden, dass Industriedatenbanken über 80 verschiedene Masterbatches mit unterschiedlichen bromhaltigen Formulierungen anbieten (SpecialChem 2019). Aufgrund dieser Mannigfaltigkeit an BFR-Typen wurde in der Fachliteratur bereits vorgeschlagen, die Grenzwerte nicht auf die gesamte Molekülmasse, sondern auf den elementaren Brom-Gesamtgehalt zu beziehen, um ein Umweltmonitoring beispielsweise mittels Röntgenfluoreszenzanalyse (RFA) durchführen zu können, da es eine relativ kostengünstige und vor allem schnell durchführbare Methode ist (z. B.: Aldrian et al. 2015; Guzzonato et al. 2016).

So gibt es bereits eine Europäische Norm von CENELEC (ÖVE/ÖNORM EN 62321-3-1: 201412 01), die die Anwendung der RFA zum Screening von Brom - wie auch von Blei, Quecksilber, Cadmium und Chrom - in „Rohstoffen, einzelnen aus Produkten entnommenen Materialien und in ,homogenisierten' Gemischen aus mehr als einem Material“ näher beschreibt. Diese Methode ist jedoch für die Grenzwertüberwachung gemäß RoHS nicht zulässig (1000 ppm für $\mathrm{PBB}$ und $1000 \mathrm{ppm}$ für PBDE), da sich diese Werte auf das gesamte Molekül und nicht nur auf den Gesamtbromgehalt beziehen. Darüber hinaus müssen die Grenzwerte gemäß Abfallbehandlungspflichtenverordnung (AbfallBPV 2017) eingehalten werden, wenn eine Verwertung von EAG-Kunststoffen außerhalb des Elektronikbereichs vorgesehen ist. Demnach ist die Einstufung als Abfall der Grünen Liste zulässig, „sofern der Bromgehalt von $2000 \mathrm{mg} / \mathrm{kg}$ im Falle von Kunststoffabfällen nachweislich unterschritten wird“ (BMNT 2017). Da hierbei der Gesamtbromgehalt nachgewiesen werden muss, wird dieser zumeist mittels RFA und nicht mittels teurer Gaschromatographie mit gekoppelter Massenspektrometrie (GC/MS) bestimmt (BMNT 2017). Hinsichtlich des Basler Übereinkommens bzw. gemäß Abfallverbringungsverordnung (Verordnung (EG) Nr. 1013/2006) ist die Ausfuhr von Abfällen, die „polybromierte Biphenyle (PBB) oder analoge polybromierte Verbindungen" in Konzentrationen von $\geq 50$ ppm enthalten, nicht erlaubt. Diese Grenzwerte sind somit um ein Vielfaches strenger, wobei die RFA hierbei wiederum nicht geeignet ist. Trotz allem hat die RFA ein großes Potenzial, sich als Nachweismethode für BFRhaltige Kunststoffe zukünftig durchzusetzen, da im Hinblick auf die in der WEEE-Richtlinie (Richtlinie (EU) 2017/19/EU; engl. Waste Electrical and Electronic Equipment) erhöhten Recyclingquoten - seit 2019 zwischen 65 und 85 Gew.-\% pro Gerätekategorie - davon ausgegangen werden kann, dass solche Stoffströme kostengünstig und in größeren Stichprobenumfängen auf BromKontaminationen verstärkt überwacht werden müssen.

Der erste Schritt, um die Diskrepanz zwischen strengen Grenzwerten und erhöhten Recyclingquoten schließen zu können, wäre Brom-belastete Kunststoffbauteile zeitnah und, wenn möglich, bereits am Anfallsort zu identifizieren und somit von Recyclingprozessen ausschließen zu können. Vor diesem Hintergrund wurden im Rahmen dieser Studie aus 11 ausgewählten EAGKategorien insgesamt 1008 Gehäusekunststoffproben auf ihren Kunststofftyp und Bromgehalt untersucht. Diese Informationen können von Interesse für Recycling-Unternehmen sein, um einerseits Gerätekategorien zu identifizieren, die einheitliche und wertvolle Kunststofftypen enthalten, oder solche, die einen hohen Bromgehalt aufweisen. Auf Basis solcher Informationen können Kunststoff-Recyclingunternehmen Maßnahmen ergreifen, um kontaminierte Gerätekategorien vom Recyclingprozess auszuschließen und somit eine verbesserte Produktqualität der Rezyklate erreichen zu können. 


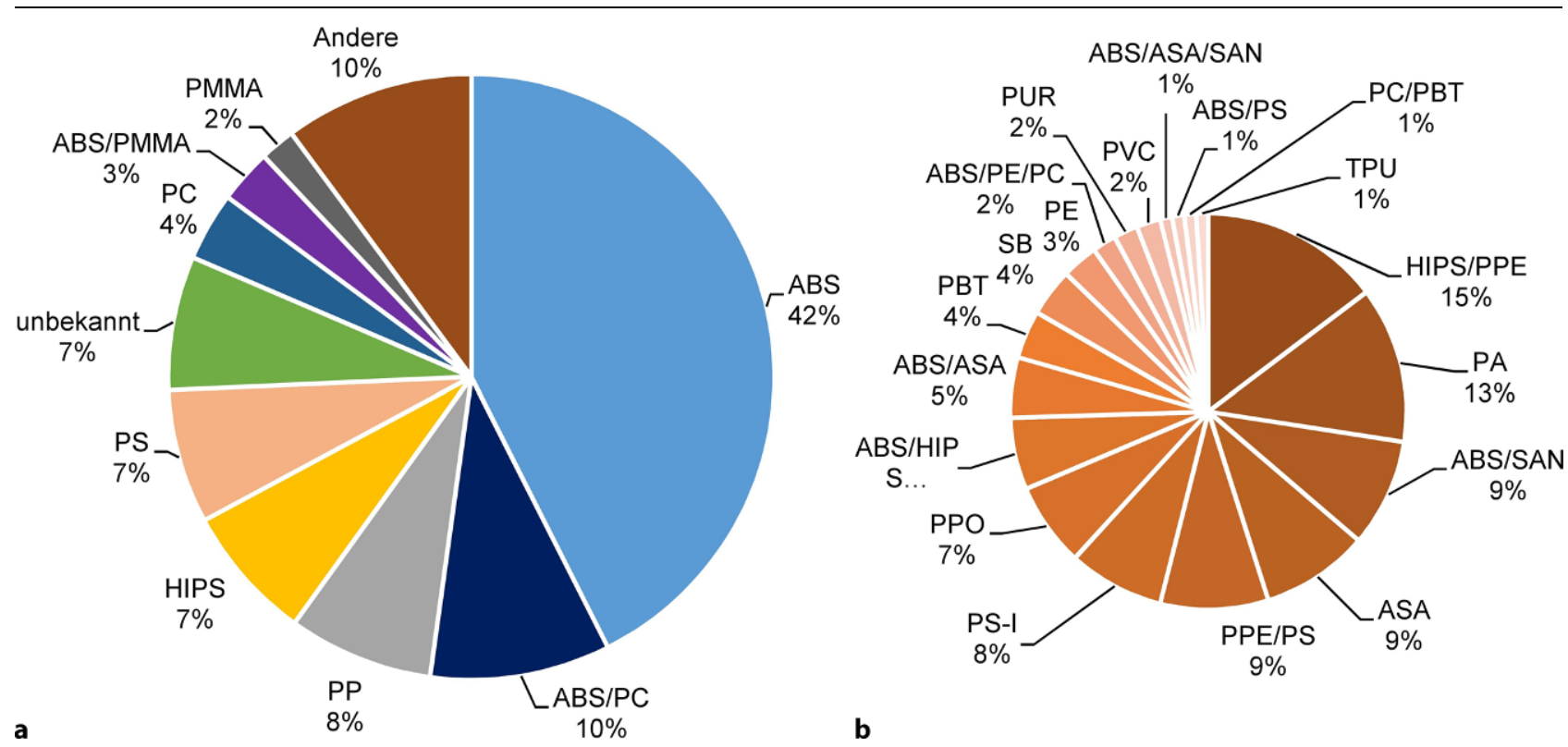

Abb. 1 Die relative Verteilung von Kunststoffsorten in EAG wurde anhand des RIC oder durch FTIR-Spektroskopie bestimmt, wobei $7 \%$ „unbekannt“ blieben bzw. nicht durch die Kunststoffdatenbank eindeutig identifiziert werden konnten. Bild (a) stellt die Anteile der am häufigsten vorkommenden Kunststoffe dar, die mit einer Häufigkeit $>1,5 \%$ vorkamen. Kunststofftypen mit einer Häufigkeit $<1,5 \%$ wurden in der Kategorie „Andere“ zusammengefasst und sind in Bild (b) näher aufgeschlüsselt

\section{Material und Methoden}

Für diese Studie wurden Geräte aus 11 EAG-Kategorien aus dem Bestand eines Wiener EAG-Recycling-Unternehmens entnommen. Die Auswahl der EAG-Kategorien wurde in Anlehnung an die Studie von Beigl und Salhofer (2016) getroffen. Bei den Gerätetypen handelt es sich um 53 PC-Tastaturen, 56 PC-Mäuse, 70 PC-Gehäuse, 70 Druckernetzteile, 70 Staubsauger, 50 elektrische Zahnbürsten, 18 Mobiltelefone ohne Touchscreen, 14 Smartphones, 10 große Haushaltselektrogeräte (5 Waschmaschinen und $5 \mathrm{Ge}$ schirrspüler), 27 Flatscreen-Monitore und 29 kleine Haushaltselektrogeräte (7 Stabmixer, 8 Toaster, 6 Wasserkocher, 6 Kaffeemaschinen, 1 Entsafter und 1 Brotschneidemaschine).

Diese Geräte wurden händisch zerlegt, in ihre funktionellen Kunststoffelemente getrennt und visuell auf den internationalen Recycling Code (engl. Resin Identification bzw. Recycling Code, kurz RIC) hin geprüft. Da grundsätzlich keine Kennzeichnungspflicht besteht und kleine Kunststoffteile ohnehin ausgenommen sind, war eine Identifikation nach RIC in vielen Fällen nicht möglich. In solchen Fällen wurden die Kunststofftypen mittels Fourier-Transform-Infrarotspektrometer (ATR-FTIR, Bruker) identifiziert. Für diesen Zweck wurde die Oberfläche der einzelnen Proben abgefeilt, um etwaige Lackierungen $\mathrm{zu}$ entfernen und den Kunststoff für eine eindeutige Identifikation freizugeben. Die Infrarotspektren wurden hierfür mit einer kommerziellen Polymerdatenbank (Biorad, Deutschland) abgeglichen.

Zur Bestimmung des Bromgehalts wurden kleine Kunststoffteile aus den Proben herausgeschnitten $\left(1 \mathrm{~cm}^{2}\right.$ bis $30 \mathrm{~cm}^{2}$ ), um eine geeignete Größe für die RFA-Messkammer zu erhalten. Die RFA-Messkammer diente vor allem der Strahlensicherheit und erlaubte auch einen konstanten Abstand zwischen Messgerät und Probe, um somit die Reproduzierbarkeit der Messungen erhöhen zu können. Jede Probe wurde drei Mal mittels portablem RFA-Spektrometer analysiert (XL3T950, Thermo Scientific Portable Analytical Instruments Inc., Tewsbury, USA). Das Analysegerät ist mit einer Goldanode ausgestattet und misst bei einer maximalen Spannung von $50 \mathrm{kV}$ und maximalen Stromstärke von $100 \mu \mathrm{A}$. Als Messzeit wurden $50 \mathrm{~s}$ je Emissionsfilter gewählt, da dadurch eine Signalsättigung des bromspezifischen Ka-Peaks bei $11,92 \mathrm{keV}$ gewährleistet werden konnte.

Zur Grenzwertüberwachung gemäß RoHS und AbfallBPV sowie zur Bestimmung der allgemeinen Nachweisgrenze für Brom wurden zwei zertifizierte Re- ferenzmaterialien herangezogen: Beim Europäischen Referenzmaterial (ERM ${ }^{\circledR}$ - „EC590“) handelt es sich um ein Granulat aus LDPE (low density Polyethylen) mit einem Durchmesser von 2 bis $3 \mathrm{~mm}$, welches aus einer technischen Mischung aus PentaBDE, OctaBDE, DecaBDE und DecaBB sowie Antimonoxid $\left(\mathrm{Sb}_{2} \mathrm{O}_{3}\right)$ besteht. Der Bromgehalt im ERM beträgt laut Analysezertifikat $2130 \pm 90 \mathrm{ppm}$. Es enthielt keine Schwermetalle oder andere Additive. Das zweite Referenzmaterial stammt von der Bundesanstalt für Materialforschung und -prüfung in Deutschland („BAM-010“) und besteht aus einer $1 \mathrm{~mm}$ dicken ABS-Scheibe mit einem Bromgehalt von $240 \pm 21 \mathrm{ppm}$. Es enthält auch Blei, Cadmium, Chrom und Quecksilber. Unter den gewählten Messbedingungen entsprachen diese Bromgehalte beim „BAM-010“ einem Messwert von 62 Zählimpulsen pro Sekunde (engl. Counts per second, kurz cps) und beim „EC590“ 746 cps. So konnten in weiterer Folge die Grenzwerte und die Nachweisgrenze bestimmt werden. Bei der portablen RFA kann im Falle von Kunststoffproben von einer Nachweisgrenze von $10 \mathrm{ppm}$ ausgegangen werden (Turner and Filella 2017a). Dieser Wert entsprach in unserer Studie wiederum $3 \mathrm{cps}$. In weiterer Folge konnte der AbfallBPV-Grenzwert von $2000 \mathrm{ppm}$ auf $700 \mathrm{cps}$ umgerech- 

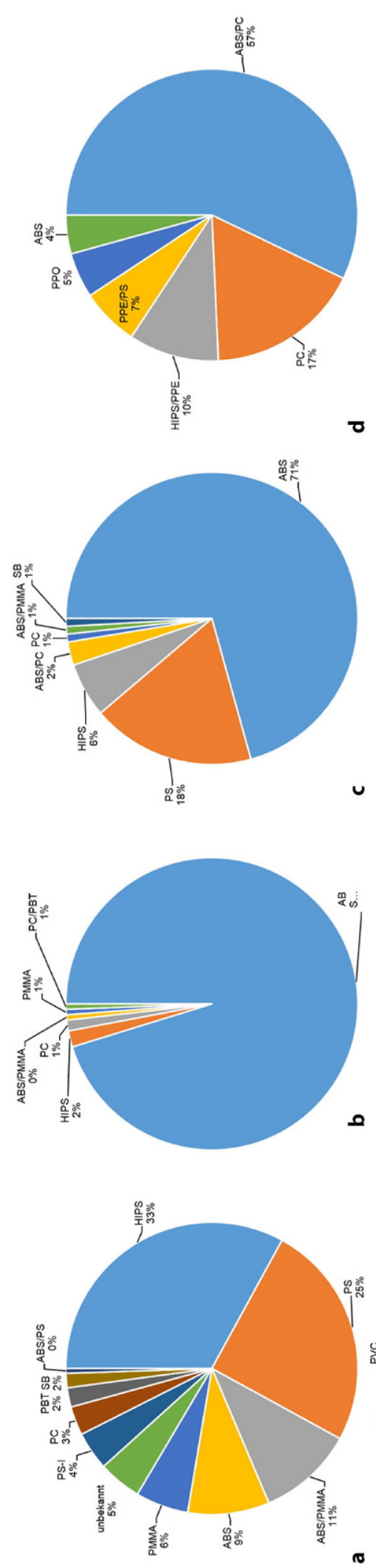
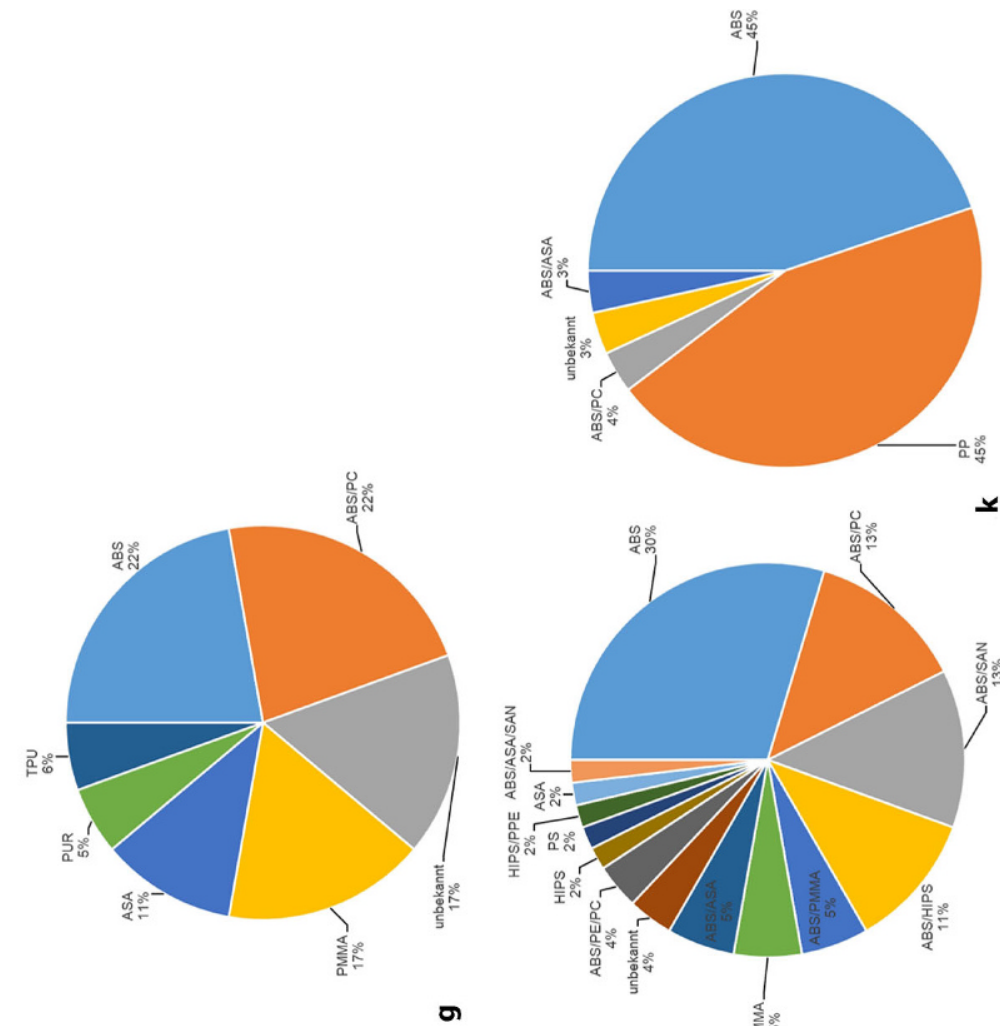

$\frac{1}{\frac{1}{D}} \frac{1}{\Phi}$

N

之

0능

ญ

:

के ठㅇ

1

के $\frac{x}{0}$

ळ

: $\sum_{1} \frac{\bar{\tau}}{\sigma}$

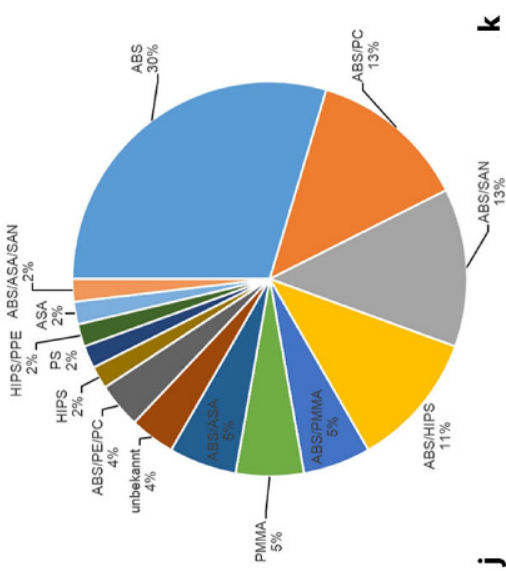

(1)

ฮั ळ

紊 임

跑

10

듬

등

ว $\frac{t}{(0)}$

ญे है

:वृ

ธิ

(1)

ํ.

ฮ 음

트으

क्ष
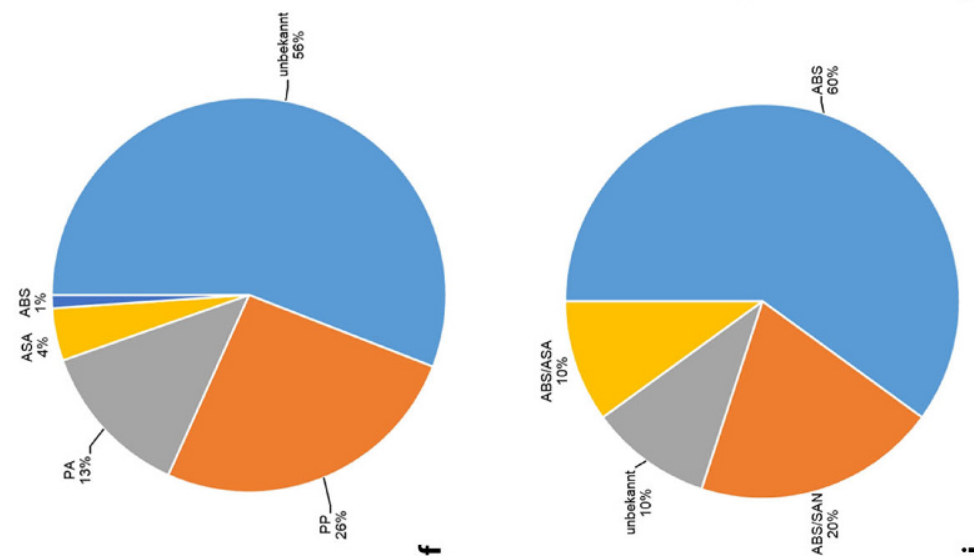

등

응

ㅎํㅇ

光 $\frac{4}{0}$

ㅎํㅇ

뜬 응

웡

त)

交离

范

岸

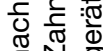

N $\mathrm{O}$

-

告依 $\frac{0}{9}$

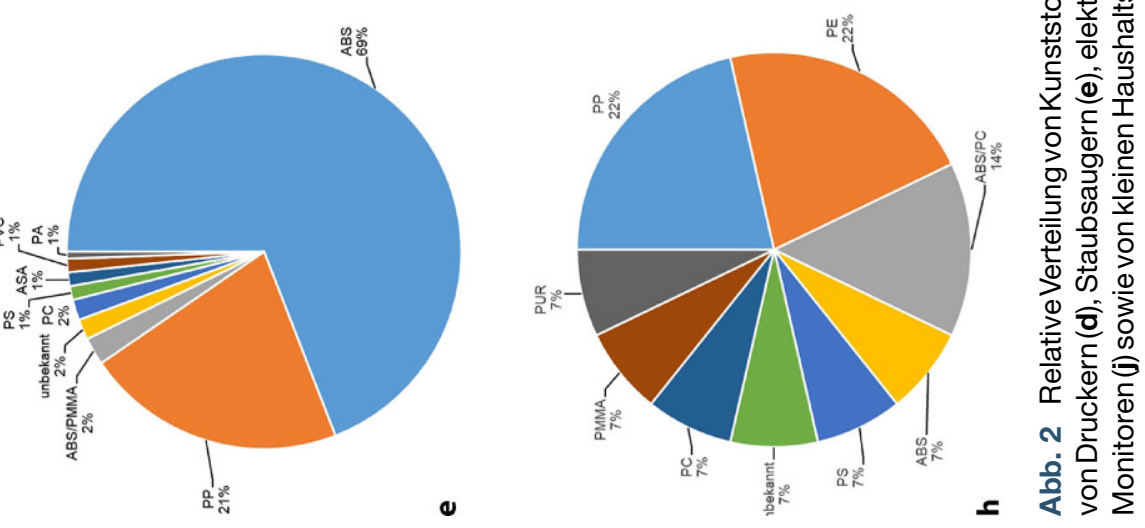




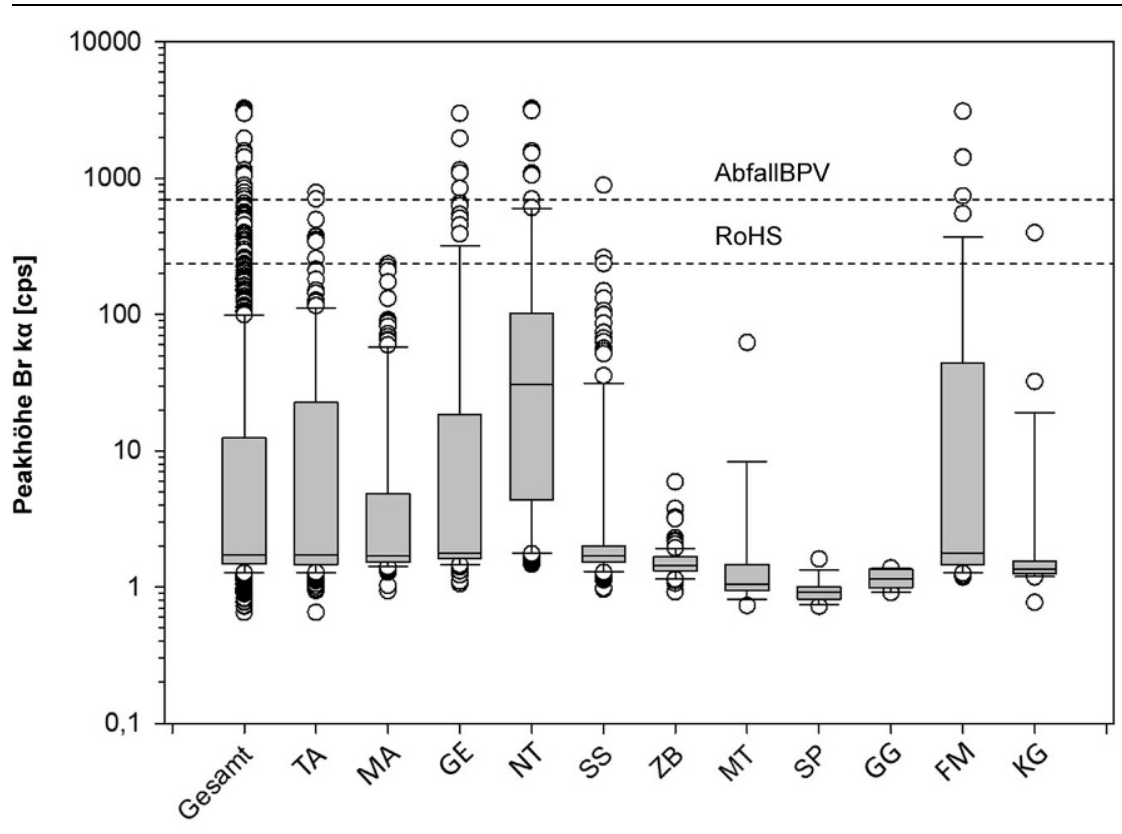

EAG-Kategorie

Abb. 3 Box-Plot-Diagramme der Bromverteilung im gesamten Probenumfang (Gesamt, $n=1008$ ) und in den einzelnenEAG-Kategorien: PC-Tastaturen (TA), -Mäuse $(M A)$, - Gehäuse (GE), Druckernetzteile (NT), Staubsauger (SS), elektrische Zahnbürsten (ZB), Mobiltelefone ohne Touchscreen (MT) Smartphones (SP), große Haushaltselektrogeräte (GG), Flatscreen-Monitore ( $F M)$ und kleine Haushaltselektrogeräte $(K G)$. Die graugefärbten Boxen sind durch das untere Quartil, den Median sowie das obere Quartil begrenzt, wobei $25 \%, 50 \%$ bzw. $75 \%$ aller Daten kleiner gleich diesen Referenzwerten liegen. Die horizontale Linie innerhalb der Box stellt den Median dar, während die Antennen (Whiskers) die Verteilung von 10 bis $90 \%$ der Messdaten um den Median anzeigen. Oberhalb und unterhalb der Antennen befinden sich Ausreißer, die als Kreise dargestellt sind. Die gestrichelten Linien entsprechen dem RoHS- und AbfallBPV-Grenzwert (umgerechnet 820 ppm fürBFR bzw. 2000 ppmfürGesamtbromgehalt). DieAsymmetrieder Boxen bzw. schiefe Lage der Mediane gibt darüber hinaus einen Hinweis darauf, dass die zugrunde liegende Verteilung der Messdaten keiner Normalverteilung unterliegt

net werden. Der RoHS-Grenzwert von 1000 ppm bezieht sich jedoch auf PBB bzw. PBDE und musste zuerst in Bromgehalte umgerechnet werden. Dies erfolgte auf Basis von Aldrian et al. (2015), wobei der RoHS-Grenzwert einem mittels RFA gemessenen Bromgehalt von $820 \mathrm{ppm}$ entspricht. In unserer Studie entsprach dieser Wert $249 \mathrm{cps}$. Die Ergebnisse der gemessenen Bromgesamtgehalte wurden mittels Box-PlotDiagrammen dargestellt.

\section{Ergebnisse}

\subsection{Identifizierte Kunststofftypen}

Mittels RIC-Screening und FTIR-Spektrometer konnten auf Basis von insgesamt 1008 Kunststoffproben, die aus 11 unterschiedlichen Gerätekategorien entnommen wurden, 27 unterschiedliche Kunststofftypen in den unter- tisches Polyurethan (TPU), Polyphenylenoxid (PPO), Styrol-Budatien (SB) Polybutylenterephthalat (PBT) etc. zu finden. Diese Ergebnisse heben somit hervor, wie mannigfaltig die stoffliche Zusammensetzung von EAG-Kunststoffen sein kann.

Die detaillierte Verteilung der Kunststoffsorten innerhalb der einzelnen EAG-Kategorien wird in Abb. 2 dargestellt und offenbart, dass einige Gerätekategorien von bestimmten Kunststofftypen dominiert werden. Gehäuse von PC-Mäusen bestanden in $95 \%$ der Fälle aus ABS (vgl. Abb. 2b). Auch bei PCGehäusen (c, 71\%), Staubsaugern (e, $69 \%)$, großen und kleinen Haushaltsgeräten (i, $60 \%$; k, 45\%), FlatscreenMonitoren (j, 30\%) und Mobiltelefonen ohne Touchscreen (g, 22\%) stellte ABS die größte Fraktion dar. Gehäuse von Druckernetzteilen setzten sich am häufigsten aus einem ABS/PC Blend (d, $57 \%)$ zusammen, während Keyboards hauptsächlich aus HIPS (a, $33 \%$ ) sowie Smartphone-Gehäuse aus PP (h, 22\%) und PE (h, 22\%) bestanden. $56 \%$ der Gehäusekomponenten von elektrischen Zahnbürsten konnten nicht eindeutig zugeordnet werden. Dabei handelte es sich hauptsächlich um elastische Kunststoffe, die als Griffstück in elektrischen Zahnbürsten verwendet werden und augenscheinlich keine Thermoplaste sind.

Auf Basis dieser Ergebnisse können detaillierte Aussagen zur Kunststoffzusammensetzung von EAG nach Gerätekategorien getroffen werden, um mittels (Vor-)Sortierprozessen homogene bzw. sortenreine Fraktionen herstellen zu können. Auf diese Weise könnten beispielsweise auch jene Gerätekategorien (z. B. Smartphones oder FlatscreenMonitore) ausgeschlossen bzw. aussortiert werden, welche sehr heterogen zusammengesetzt sind und somit unter sehr hohem Energie- und Kosteneinsatz aufgetrennt werden müssten.

\subsection{Bromgehalt nach EAG-Kategorie}

Die untersuchten Kunststoffproben wurden für jede EAG-Kategorie mittels RFA auf ihren Bromgehalt näher untersucht. Die Ergebnisse sind in Abb. 3 näher dargestellt. In Tab. 1 wurden die Gerätekategorien in Hinblick auf Grenzwertüberschreitungen näher ausgewertet. Die Auswertung der RFADaten hat ergeben, dass in ca. $33 \%$ aller Proben Brom nachgewiesen werden konnte. Mit anderen Worten lag hier der 
Tab. 1 Nachweisgrenze (NG), Grenzwertüberschreitungen gemäß RoHS und AbfallBPV sowie Mittelwerte der mittels RFA gemessenen Bromgesamtgehalte der EAG-Kategorien: PC-Tastaturen (TA), -Mäuse (MA), -Gehäuse (GE), Druckernetzteile (NT), Staubsauger (SS), elektrischeZahnbürsten (ZB), Mobiltelefone ohne Touchscreen (MT) Smartphones (SP), große Haushaltselektrogeräte (GG), Flatscreen-Monitore (FM) und kleine Haushaltselektrogeräte $(K G)$

\begin{tabular}{|c|c|c|c|c|c|c|c|c|c|}
\hline \multicolumn{2}{|c|}{ EAG-Kategoric Anzahl } & \multicolumn{6}{|c|}{ Grenzwertüberschreitung } & \multicolumn{2}{|c|}{ Mittlerer Brom- } \\
\hline & & \multicolumn{2}{|c|}{$>\mathrm{NG}$} & \multicolumn{2}{|c|}{$>\mathrm{RoHS}$} & \multicolumn{2}{|c|}{$>$ AbfallBPV } & \multirow[b]{2}{*}{ [cps] } & \multirow[b]{2}{*}{ [ppm] } \\
\hline & {$[n]$} & {$[n]$} & [\%] & {$[n]$} & {$[\%]$} & {$[n]$} & {$[\%]$} & & \\
\hline Alle & 1008 & 337 & 33,4 & 53 & 5,3 & 25 & 2,5 & 70,9 & 236,2 \\
\hline TA & 188 & 70 & 37,2 & 8 & 4,3 & 2 & 1,1 & 39,9 & 132,9 \\
\hline MA & 168 & 51 & 30,4 & 0 & 0,0 & 0 & 0,0 & 15,8 & 52,5 \\
\hline GE & 116 & 38 & 32,8 & 12 & 10,3 & 5 & 4,3 & 115,3 & 384,2 \\
\hline NT & 140 & 111 & 79,3 & 25 & 17,9 & 13 & 9,3 & 257,1 & 857,1 \\
\hline SS & 178 & 37 & 20,8 & 2 & 1,1 & 1 & 0,6 & 16,9 & 56,4 \\
\hline ZB & 93 & 4 & 4,3 & 0 & 0,0 & 0 & 0,0 & 1,6 & 5,3 \\
\hline MT & 18 & 1 & 5,6 & 0 & 0,0 & 0 & 0,0 & 4,6 & 15,2 \\
\hline $\mathrm{SP}$ & 14 & 0 & 0,0 & 0 & 0,0 & 0 & 0,0 & 0,9 & 3,2 \\
\hline GG & 10 & 0 & 0,0 & 0 & 0,0 & 0 & 0,0 & 1,2 & 3,9 \\
\hline FM & 54 & 22 & 40,7 & 5 & 9,3 & 4 & 7,4 & 43,7 & 145,5 \\
\hline$K G$ & 29 & 3 & 10,3 & 1 & 3,4 & 0 & 0,0 & 16,7 & 55,6 \\
\hline
\end{tabular}

Bromgesamtgehalt über der Nachweisgrenze von $10 \mathrm{ppm}$. Im Vergleich dazu wurde in Frankreich eine umfassende Studie an 347 EAG durchgeführt, wobei bei $46 \%$ der untersuchten Geräte in zumindest einem Bauteil Brom mittels RFA nachgewiesen wurde (Hennebert und Filella 2018).

In Tab. 1 ist ebenso ersichtlich, dass bei 5,3\% aller Proben der RoHS-Grenzwert und bei $2,5 \%$ der Grenzwert gemäß AbfallBPV überschritten wurde. Das arithmetische Mittel des Gesamtbromgehalts aller Kunststoffproben betrug $237 \mathrm{ppm}$. Somit würden alle Kategorien im Durchschnitt unter den Grenzwerten gemäß RoHS sowie $\mathrm{Ab}$ fallBPV liegen, aber dennoch über dem Grenzwert gemäß Abfallverbringungsverordnung (ca. 4,7-fach überschritten). Im Vergleich zum arithmetischen Mittel lag der Median aller Proben mit Ausnahme von Netzteilen unter der Nachweisgrenze. Die Box-Plot-Darstellung (siehe Abb. 3) zeigt die Verteilung des Bromgehalts innerhalb der 1008 Proben (Gesamt). Die graue Box umfasst $50 \%$ aller Proben, die dem Median am nächsten sind, während die Antennen (Whisker) $80 \%$ der Verteilung abdecken. Ausreißer ober- und unterhalb der Antennen werden durch Kreise dargestellt. Mit anderen Worten wurde der untersuchte EAG-Strom durch wenige Ausreißer bzw. durch Proben mit hohen Bromgehalten verunreinigt. Solche Ausreißer könnten zukünftig mittels RFA vor Ort zeitnah identifiziert und vorzeitig aussortiert werden.
Die Detailanalyse der einzelnen EAG-Kategorien hat gezeigt, dass Druckernetzteile die höchsten Bromgehalte aufwiesen. In ca. $79 \%$ dieser Proben konnte Brom nachgewiesen werden, wobei ca. $18 \%$ aller Proben über dem RoHS- und 9\% über dem AbfallBPVGrenzwert lagen. Im Mittel wies diese Kategorie eine Bromkonzentration von $857 \mathrm{ppm}$ auf und lag somit über dem umgerechneten RoHS-Grenzwert von $820 \mathrm{ppm}$. Ca. 9\% der Proben der Kategorien Flatscreen-Monitore und $10 \%$ der PC-Gehäuse überschreiten den RoHS-Grenzwert. Neben diesen Kategorien überschreiten auch PCTastaturen, PC-Gehäuse, Staubsauger und kleine Haushaltsgeräte teilweise die Grenzwerte gemäß RoHS und AbfallBPV. Im Gegensatz dazu wurde der RoHS-Grenzwert in PC-Mäusen, elektrischen Zahnbürsten, Mobiltelefonen (ohne Touchscreen), Smartphones und großen Haushaltgeräten in allen Fällen eingehalten. In den Kategorien Smartphones und große Haushaltsgeräte (Waschmaschinen und Geschirrspüler) konnte kein Brom nachgewiesen werden. Dies deutet daraufhin, dass in diese Gerätekategorien vorwiegend bromfreie FSM und wahrscheinlich auch keine mit Brom belastete Rezyklate eingearbeitet wurden.

\subsection{Bromgehalt nach Kunststofftyp}

Die Detailanalyse des Bromgehalts nach Kunststofftyp hat ergeben (siehe Abb. 4), dass nur 6 der bekannten Kunststofftypen sowie eine unbekannte
Kunststoffprobe den RoHS-Grenzwert überschritten haben. Diese Kunststofftypen weisen auch breite Verteilungen der Messdaten auf, die darauf hindeuten, dass der Bromgehalt sowohl sehr gering als auch sehr hoch sein kann. Bemerkenswert dabei ist, dass die Überschreitung des RoHS-Grenzwerts in 7 Gerätekategorien vorgefunden wurde. Das deutet darauf hin, dass nur in bestimmten Kunststofftypen mit hohen Brom-Mengen zu rechnen ist und diese unabhängig vom Gerätetyp sind.

Die häufigsten Überschreitungen des RoHS-Grenzwertes kamen in Kunststofftypen PC (19\%), PS (18\%), ABS/PC (14\%), ABS/PMMA (4\%), ABS (3\%) und $1 \%$ der unbekannten Kunststofftypen vor. Insgesamt überschritten $5 \%$ aller Proben den RoHS- und 2\% den AbfallBPV-Grenzwert. Überschreitungen über $10 \%$ des AbfallBPV-Grenzwerts wurden bei ABS/HIPS (17\%) und PC (14\%) festgestellt. Diese sind die zwei am häufigsten in Druckernetzteilen eingesetzten Kunststoffe - jene EAG-Kategorie mit dem höchsten Bromgehalt (vgl. mit Abschn. 3.1.). In PS (6\%), ABS/PC (4\%), ABS/PMMA (3\%), ABS (2\%) und den unbekannten Kunststoffen (1\%) wurde der AbfallBPV-Grenzwert seltener überschritten.

\subsection{Bromgehalt in Abhängigkeit vom Produktionsjahr}

In $38 \%$ der analysierten Kunststoffproben war eine visuelle Bestimmung des Produktionsdatums möglich. In Abb. 5 wird das Produktionsdatum in Relation 


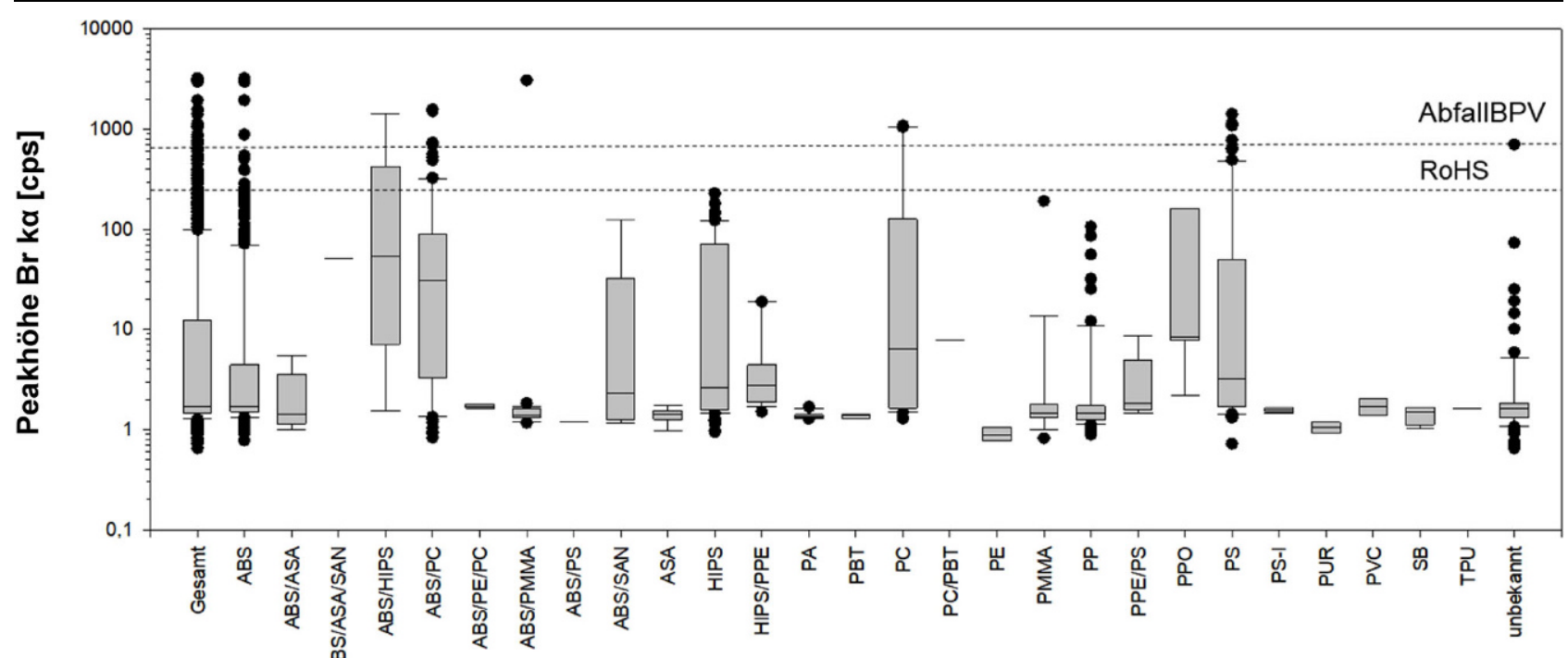

a

Kunststofftyp

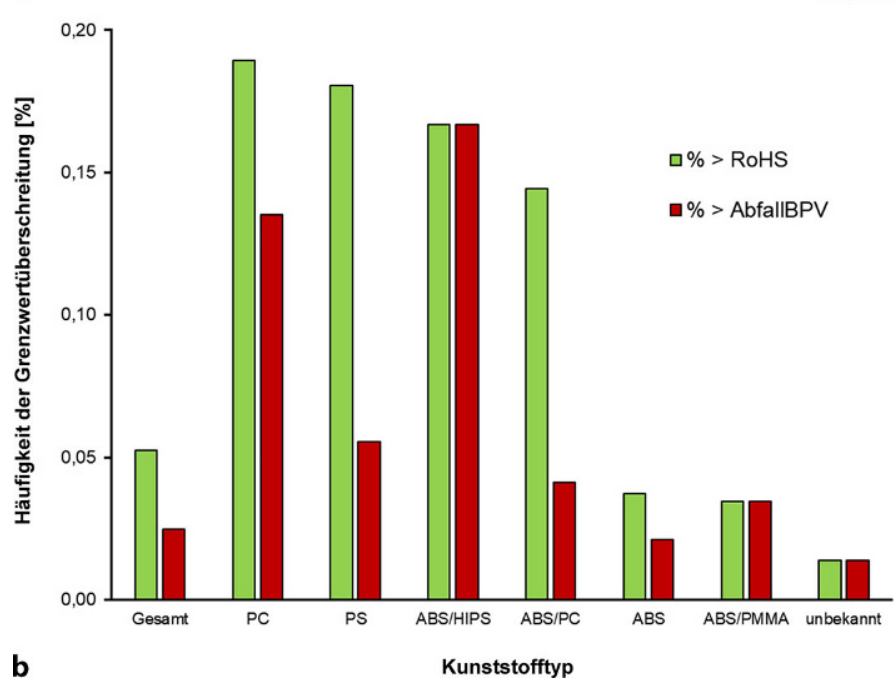

Abb. 4 Verteilung des Gesamtbromgehaltsund Überschreitung derGrenzwerteinAbhängigkeitvom Kunststofftyp. Bild (a)zeigtanhand von Box-Plot-Diagrammen die Bromverteilung im gesamten Probenumfang (Gesamt) sowie in den einzelnen Kunststofftypen, die in den untersuchten EAG-Kategorien gefunden wurden. Die grauen Boxen stellen die Verteilung von 25 bis $75 \%$ aller Messdaten und die horizontalen Linien innerhalb der Boxen den Median dar. Die Antennen (Whisker) stellen die Verteilung von 10 bis $90 \%$ aller Messdaten um den Median dar. Oberhalb und unterhalb der Whisker sind die Ausreißer der Messdaten als Kreise abgebildet. Die gestrichelten horizontalen Linien stellen die in cps umgerechneten Grenzwerte gemäß RoHS und AbfallBPV zur Orientierung dar. In Bild (b) ist der prozentuelle Anteil jener Kunststofftypen ersichtlich, der über dem RoHS- (grün) oder AbfallBPV-Grenzwert (rot) liegen. In diesem Bild sind somit jene 20 Kunststofftypen nicht enthalten, in denen die Grenzwerte unterschritten wurden

zum gemessenen Bromgehalt gestellt. Es ist erkennbar, dass der Bromgehalt in EAG, die nach 2005 produziert wurden, tendenziell abnahm. Die RFADatenauswertung hat auch ergeben, dass ab 2011 alle der analysierten Proben sowohl den AbfallBPV- als auch den RoHS-Grenzwert unterschreiten. Erwähnenswert ist, dass der AbfallBPVGrenzwert nur von wenigen Proben im betrachteten Zeitraum überschritten wurde. Im Gegensatz dazu wird der RoHS-Grenzwert von vielen Proben bis zum Produktionsjahr 2010 überschrit- ten. Diese Daten deuten somit darauf hin, dass bromierte FSM bereits durch halogenfreie FSM substituiert wurden, oder dass der Bromgesamtgehalt durch den Einsatz von Synergisten, wie Antimon(III)-oxid $\left(\mathrm{Sb}_{2} \mathrm{O}_{3}\right)$, wesentlich reduziert werden konnte. In der Studie von Hennebert und Filella (2018) wurde ebenso eine zeitliche Abnahme der Bromgehalts festgestellt. In weiteren Studien aus Großbritannien und der Schweiz konnte mittels RFA nachgewiesen werden, dass vor allem in EAGBauteilen antimonhaltige Synergisten in Begleitung mit Brom gefunden wurden (Turner and Filella 2017a, 2017b).

\section{Diskussion und Schlussfolgerung}

Die augenscheinliche Untersuchung des RICs in Kombination mit FTIRSpektroskopie hat gezeigt, dass die Kunststoffzusammensetzung von EAG sehr heterogen ist, da 27 unterschiedliche Kunststofftypen bzw. Polymerkombinationen identifiziert werden konnten. Bemerkenswert ist, dass lediglich 8 Kunststofftypen und -blends über 


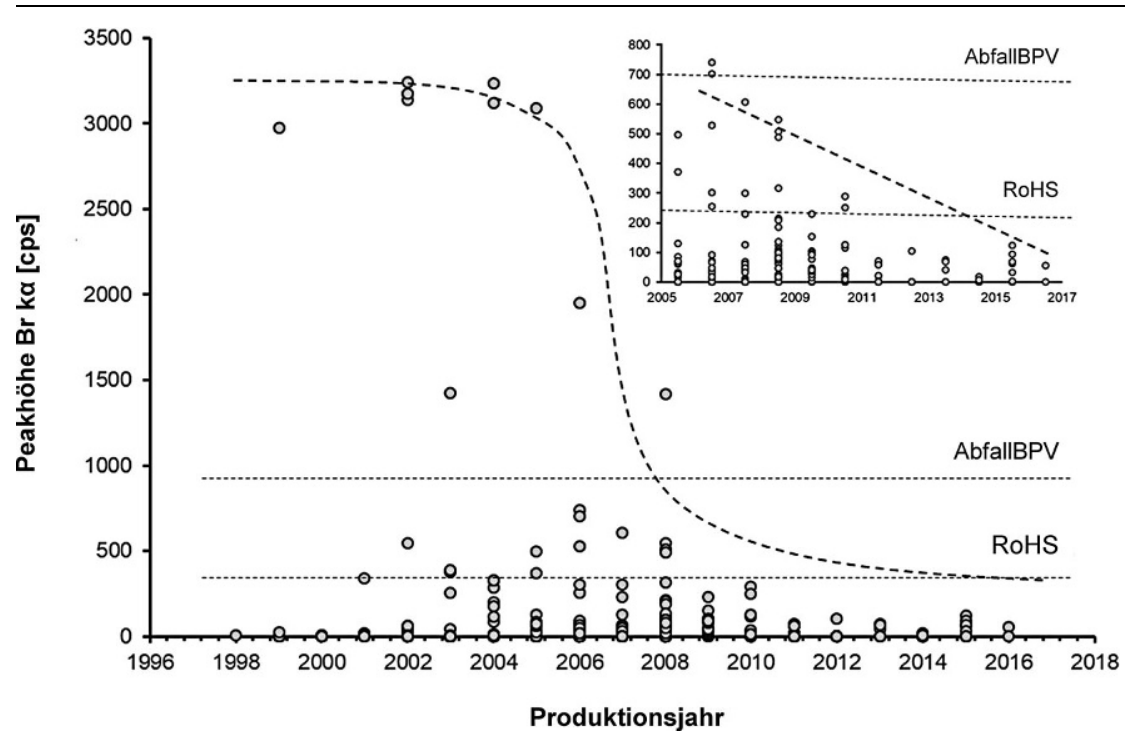

Abb. 5 Gesamtbromgehalt in EAG in Abhängigkeit vom Produktionsjahr. Die dick gestrichelten Linien dienen ausschließlich der stark vereinfachten Darstellung des Trendverlaufs (statistisch nicht abgesichert). In der rechten oberen Ecke ist der zeitliche Ausschnitt von 2005 bis 2017 des EAG-Bromgehalts dargestellt. Die horizontal verlaufenden, dünn gestrichelten Linien entsprechen den in cps umgerechneten Grenzwerten gemäß RoHS und AbfallBPV

$80 \%$ aller in EAG eingesetzten Kunststofftypen ausmachen.

Die durchgeführte RFA ermöglichte es darüber hinaus, den Bromgesamtgehalt auf einfache Weise in einem großen Stichprobenumfang zu quantifizieren. Hierbei konnte dank zertifizierter Referenzmaterialien eine ausreichende Genauigkeit des Messverfahrens erzielt werden. Aus 11 unterschiedlichen Gerätekategorien wurden 467 EAGProben gezogen, aus welchen wiederum insgesamt 1008 Kunststoffproben entnommen wurden. Ein nachweisbarer Bromgehalt wurde in $33,4 \%$ aller Proben festgestellt, wobei bei $5,3 \%$ aller Proben der RoHS- und bei 2,5\% der AbfallBPV-Grenzwert überschritten wurde. Ähnliche Studien konnten ebenso aufzeigen, dass Brom sehr heterogen in bestimmten EAG-Kategorien sowie -Bauteilen verteilt sein kann (vergleiche z.B. mit Hennenbert und Filella 2018; Turner und Filella 2017a). So konnte in dieser Studie nachgewiesen werden, dass in Netzteilen von Druckern der höchste Bromgehalt vorlag, wobei in
$79 \%$ dieser Kunststoffproben Brom detektiert werden konnte und bei $18 \%$ der RoHS-Grenzwert überschritten wurde. Des Weiteren wurde Brom in rund $41 \%$ aller Proben aus Flatscreen-Monitoren, in $37 \%$ aus PC-Tastaturen, in $33 \%$ aus PC-Gehäusen, in $30 \%$ aus PC-Mäusen sowie in $21 \%$ aller Proben aus Staubsaugern mittels RFA nachgewiesen. Der Gesamtbromgehalt lag bei $10 \%$ aller Proben aus Kleingeräten (Stabmixer, Toaster etc.), bei $6 \%$ aus Mobiltelefonen (ohne Touchscreen) und bei $4 \%$ aus elektrischen Zahnbürsten über der Nachweisgrenze. In den untersuchten Smartphones und Großgeräten (Waschmaschinen und Geschirrspüler) wurde kein Brom gefunden.

Für alle untersuchten EAG-Kategorien konnte ein durchschnittlicher Gesamtbromgehalt von ca. $240 \mathrm{ppm}$ bestimmt werden. Dieser Gehalt ist laut Fachliteratur (vergleiche z.B. mit Alaee et al. 2003; Arias 2001; Bureau 2003) jedoch nicht ausreichend, um die Mindestanforderungen für eine effektive Flammschutzwirkung in Kunst- stoffen gewährleisten zu können. Die Ergebnisse deuten auch darauf hin, dass EAG-Kunststoffe bereits bromfreie FSM (z.B. aus aluminium-, magnesium- oder phosphorhaltigen Verbindungen) enthalten. In Anlehnung an die Fachliteratur (vergleiche z.B. mit Turner 2018; Turner und Filella 2017a) wird auch darauf hingewiesen, dass die stoffliche Verwertung von bromhaltigen Kunststoffen aus EAG zu einer Kreuzkontamination führen kann, wobei halogenierte Substanzen unbeabsichtigt in anderswertige Kunststoffprodukte transferiert werden können.

Durch solche Detailanalysen wird es ermöglicht, bestimmte Gerätekategorien oder Kunststoffbauteile mit relativ hohem Bromgehalt anhand von RFASchnellanalysen direkt am Anfallort vorzeitig auszuschließen bzw. auszusortieren. Die RFA bietet eine kostengünstige und vor allem sehr schnelle Alternative zu konventionellen Analysemethoden, wie GC/MS, und ermöglicht es somit einen sehr großen Stichprobenumfang zu beproben. Das Analyseverfahren - vom Probensampling bis zur Datenauswertung und -interpretation müsste jedoch zukünftig auf nationaler, europäischer und/oder internationaler Ebene standardisiert werden, um die RFA zur Grenzwertüberwachung von diversen Kunststoffabfällen etablieren zu können.

Funding Open access funding provided by University of Natural Resources and Life Sciences Vienna (BOKU).

Open Access Dieser Artikel wird unter der Creative Commons Namensnennung 4.0 International Lizenz (http:// creativecommons.org/licenses/by/4. $0 /$ deed.de) veröffentlicht, welche die Nutzung, Vervielfältigung, Bearbeitung, Verbreitung und Wiedergabe in jeglichem Medium und Format erlaubt, sofern Sie den/die ursprünglichen $\mathrm{Au}$ tor(en) und die Quelle ordnungsgemäß nennen, einen Link zur Creative Commons Lizenz beifügen und angeben, ob Änderungen vorgenommen wurden. 
AbfallBPV (2017): Verordnung des Bundesministers für Land- und Forstwirtschaft, Umwelt und Wasserwirtschaft über Abfallbehandlungspflichten (AbfallBPV). StF: BGBl. II Nr. 102/2017. Achilias, D., Antonakou, E. (2015): Chemical and Thermochemical Recycling of Polymers from Waste Electrical and Electronic Equipment. Alaee, M., Arias, P., Sjödin, A., Bergman, Å. (2003): An overview of commercially used brominated flame retardants, their applications, their use patterns in different countries/regions and possible modes of release. Environmen International 29, 683-689, https://doi.org/10. 1016/S0160-4120(03)00121-1.

Aldrian, A., Ledersteger, A., Pomberger, R. (2015): Monitoring of WEEE plastics in regards to brominated flame retardants using handheld XRF. Waste Management 36, 297-304, https:// doi.org/10.1016/j.wasman.2014.10.025.

Arias, P.A. (2001): Brominated flame retardant-an overview. Proc. 2nd int. workshop on brominated flame retardants, Stockholm, Sweden, 17-19.

Beach, M.W., Hull, J.W., King, B.A., Beulich, I.I., Stobby, B.G., Kram, S.L., Gorman, D.B. (2017): Development of a new class of brominated polymeric flame retardants based on copolymers of styrene and polybutadiene. Polymer Degradation and Stability 135, 99-110, https://doi.org/10. 1016/j.polymdegradstab.2016.11.008.

Beigl, P., Salhofer, S. (2016):. Investigation of the composition of small electrical appliances and development of a dismantling database (in German), Tagungsband zur 13. Recy \& DepoTech Konferenz, in: Pomberger, R., Adam, J., Aldrian, A., Hiden, S., Höllen, D., Kranzinger, L., Lorber, K.E., Neuhold, S., Nigl, T., Pfandl, K., Sarc, R. Schwarz, T., Sedlazeck, P., Wellacher, M. (Eds.), DepoTech 2016, Montanuniversität Leoben/ Austria.

BMNT (2017): Bundesabfallwirtschaftsplan Teil 2. Bundesministeriums für Nachhaltigkeit und Tourismus (BMNT), Stubenring 1, 1010 Wien.

Buczko, A., Stelzig, T., Bommer, L., Rentsch, D., Heneczkowski, M., Gaan, S. (2014): Bridged DOPO derivatives as flame retardants for PA6. Polymer Degradation and Stability 107, 158-165, https://doi.org/10.1016/j.polymdegradstab. 2014.05.017.

Bureau, E.C. (2003): European Union Risk Assessment Report-DIPHENYL ETHER, OCTABROMO DERIVATIVE.
EC 1907/2006, Regulation (EC) No 1907/2006 of the European Parliament and of the Council of 18 December 2006 concerning the Registration, Evaluation, Authorisation and $\mathrm{Re}-$ striction of Chemicals (REACH), establishing a European Chemicals Agency, amending Directive 1999/45/EC and repealing Council Regulation (EEC) No 793/93 and Commission Regulation (EC) No 1488/94 as well as Council Directive 76/769/EEC and Commission Directives 91/155/EEC, 93/67/EEC, 93/105/EC and 2000/21/EC.

Guzzonato, A., Puype, F., Harrad, S.J. (2016): Improving the accuracy of hand-held X-ray fluorescence spectrometers as a tool for monitoring brominated flame retardants in waste polymers. Chemosphere 159, 89-95, https://doi.org/10 1016/j.chemosphere.2016.05.086.

Hahladakis, J.N., Velis, C.A., Weber, R., Iacovidou, E., Purnell, P. (2018): An overview of chemical additives present in plastics: Migration, release, fate and environmental impact during their use, disposal and recycling. Journal of Hazardous Materials 344, 179-199, https:// doi.org/10.1016/j.jhazmat.2017.10.014.

Hennebert, P., Filella, M. (2018): WEEE plastic sorting for bromine essential to enforce EU regulation. Waste Management 71, 390-399, https:// doi.org/10.1016/j.wasman.2017.09.031.

Kim, Y.R., Harden, F.A., Toms, L.-M.L., Norman R.E. (2014): Health consequences of exposure to brominated flame retardants: A systematic review. Chemosphere 106, 1-19, https://doi.org/ 10.1016/j.chemosphere.2013.12.064.

ÖVE/ÖNORM EN 62321-3-1: 201412 01, Verfahren zur Bestimmung von bestimmten Substanzen in Produkten der Elektrotechnik - Teil 3-1: Screening - Blei, Quecksilber, Cadmium, Gesamtchrom und Gesamtbrom durch Röntgenfluoreszenz-Spektrometrie (IEC 62321-31:2013) (deutsche Fassung). Austrian Standards Richtlinie (EU) 2017/19/EU, RICHTLINIE 2012/ 19/EU DES EUROPÄISCHEN PARLAMENTS UND DES RATES vom 4. Juli 2012 über Elektround Elektronik-Altgeräte (Neufassung).

Richtlinie 2003/11/EG, RICHTLINIE 2003/11/EG DES EUROPÄISCHEN PARLAMENTS UND DES RATES vom 6. Februar 2003 zur 24. Änderung der Richtlinie 76/769/EWG des Rates über Beschränkungen des Inverkehrbringens und der Verwendung gewisser gefährlicher Stoffe und Zubereitungen (Pentabromdiphenylether, Octabromdiphenylether).
Richtlinie 201 1/65/EU, DIRECTIVE 2011/65/EU OF THE EUROPEAN PARLIAMENT AND OF THE COUNCIL of 8 June 2011 on the restriction of the use of certain hazardous substances in electrical and electronic equipment.

SpecialChem (2019): The material selection platform. Searched by the keywords "brominated flame retardants". https://polymeradditives.specialchem.com/selectors? $\mathrm{q}=\% 20 \mathrm{bro}$ minated\%20flame\%20retardants (last access on 18.10.2019).

Stenvall, E., Tostar, S., Boldizar, A., Foreman, M.R.S., Möller, K. (2013): An analysis of the composition and metal contamination of plastics from waste electrical and electronic equipment (WEEE). Waste Management 33, 915-922, https://doi.org/10.1016/j.wasman.2012.12.022.

Turner, A. (2018): Concentrations and Migratabilities of Hazardous Elements in Second-Hand Children's Plastic toys. Environmental Science \& Technology 52, 3110-3116, https://doi.org/10. 1021/acs.est.7b04685.

Turner, A., Filella, M. (2017a): Bromine in plastic consumer products-Evidence for the widespread recycling of electronic waste. Science of The Total Environment 601-602, 374-379, https://doi.org/10.1016/j.scitotenv.2017.05.173. Turner, A., Filella, M. (2017b): Field-portableXRF reveals the ubiquity of antimony in plastic consumer products. Science of The Total Environment 584-585, 982-989, https://doi.org/10. 1016/j.scitotenv.2017.01.149.

Verordnung (EG) Nr. 1013/2006, VERORDNUNG (EG) Nr. 1013/2006 DES EUROPÄISCHEN PARLAMENTS UND DES RATES vom (14. Juni 2006) über die Verbringung von Abfällen.

Zhang, W., Li, X., Guo, X., Yang, R. (2010): Mechanical and thermal properties and flame retardancy of phosphorus-containing polyhedral oligomeric silsesquioxane (DOPO-POSS)/ polycarbonate composites. Polymer Degradation and Stability 95, 2541-2546, https://doi.org/ 10.1016/j.polymdegradstab.2010.07.036.

Hinweis des Verlags Der Verlag bleibt in Hinblick auf geografische Zuordnungen und Gebietsbezeichnungen in veröffentlichten Karten und Institutsadressen neutral. 